\title{
Block Hybrid Collocation Method with Application to Fourth Order Differential Equations
}

\author{
Lee Ken Yap ${ }^{1,2}$ and Fudziah Ismail ${ }^{2}$ \\ ${ }^{1}$ Department of Mathematical and Actuarial Sciences, Universiti Tunku Abdul Rahman, Setapak, 53300 Kuala Lumpur, Malaysia \\ ${ }^{2}$ Department of Mathematics and Institute for Mathematical Research, Universiti Putra Malaysia (UPM), \\ 43400 Serdang, Selangor, Malaysia
}

Correspondence should be addressed to Lee Ken Yap; lkyap@utar.edu.my

Received 10 July 2014; Revised 22 November 2014; Accepted 25 November 2014

Academic Editor: María Isabel Herreros

Copyright $(2015$ L. K. Yap and F. Ismail. This is an open access article distributed under the Creative Commons Attribution License, which permits unrestricted use, distribution, and reproduction in any medium, provided the original work is properly cited.

The block hybrid collocation method with three off-step points is proposed for the direct solution of fourth order ordinary differential equations. The interpolation and collocation techniques are applied on basic polynomial to generate the main and additional methods. These methods are implemented in block form to obtain the approximation at seven points simultaneously. Numerical experiments are conducted to illustrate the efficiency of the method. The method is also applied to solve the fourth order problem from ship dynamics.

\section{Introduction}

Fourth order ordinary differential equations (ODEs) arise in several fields such as fluid dynamics (see [1]), beam theory (see $[2,3]$ ), electric circuits (see [4]), ship dynamics (see [5-7]), and neural networks (see [8]). Therefore, many theoretical and numerical studies dealing with such equations have appeared in the literature.

Here, we consider general fourth order ordinary differential equations:

$$
y^{(4)}=f\left(t, y, y^{\prime}, y^{\prime \prime}, y^{\prime \prime \prime}\right)
$$

with the initial conditions

$$
\begin{gathered}
y(a)=y_{0}, \quad y^{\prime}(a)=y_{0}^{\prime}, \\
y^{\prime \prime}(a)=y_{0}^{\prime \prime}, \quad y^{\prime \prime \prime}(a)=y_{0}^{\prime \prime \prime}, \quad t \in[a, b] .
\end{gathered}
$$

Conventionally, fourth order problems (1) are reduced to system of first order ODEs and solved with the methods available in the literature. Many investigators $[2,9,10]$ remarked the drawback of this approach as it requires heavier computational work and longer execution time. Thus, the direct approach on higher order ODEs has attracted considerable attention.
Recent developments have led to the implementation of collocation method for the direct solution of fourth order ODEs (1). Awoyemi [9] proposed a multiderivative collocation method to obtain the approximation of $y$ at $t_{n+4}$. Moreover, Kayode [11, 12] developed collocation methods for the approximation of $y$ at $t_{n+5}$ with the predictor of orders five and six, respectively. These schemes $[9,11,12]$ are implemented in predictor-corrector mode with the employment of Taylor series expansions for the computation of starting values. Jator [2] remarked that the implementation of these schemes is more costly since the subroutines for incorporating the starting values lead to lengthy computational time. Thus, some attempts have been made on the self-starting collocation method which eliminates the requirement of either predictors or starting values from other methods. Jator [2] derived a collocation multistep method and used it to generate a new self-starting finite difference method. On the other hand, Olabode and Alabi [13] developed a self-starting direct block method for the approximation of $y$ at $t_{n+j}, j=$ $1,2,3,4$.

Here, we are going to derive a block hybrid collocation method for the direct solution of general fourth order ODEs (1). The method is extended from the line proposed by Jator [14] and Yap et al. [15]. We apply the interpolation and 
collocation technique on basic polynomials to derive the main and additional methods which are combined and used as block hybrid collocation method. This method generates the approximation of $y$ at four main points and three off-step points concurrently.

\section{Derivation of Block Hybrid Collocation Methods}

The hybrid collocation method that generates the approximations to the general fourth order ODEs (1) is defined as follows:

$$
\begin{aligned}
& \sum_{j=0}^{k} \alpha_{j} y_{n+j}+\sum_{j=1}^{3} \alpha_{v_{j}} y_{n+v_{j}} \\
& =h^{4}\left(\sum_{j=0}^{k} \beta_{j} f_{n+j}+\sum_{j=1}^{3} \beta_{v_{j}} f_{n+v_{j}}\right) .
\end{aligned}
$$

We approximate the solution by considering the interpolating function

$$
Y(t)=\sum_{j=0}^{r+s-1} \phi_{j} t^{j}
$$

where $t \in[a, b], \phi_{j}$ are unknown coefficients to be determined, $r$ is the number of interpolations for $4 \leq r \leq k$, and $s$ is the number of distinct collocation points with $s>0$. The continuous approximation is constructed by imposing the conditions as follows:

$$
\begin{gathered}
Y\left(t_{n+j}\right)=y_{n+j}, \quad j=0,1,2, \ldots, r-1, \\
Y^{(4)}\left(t_{n+\mu}\right)=f_{n+\mu}, \quad \mu=\left\{j, \nu_{1}, v_{2}, v_{3}\right\}, \quad j=0,1,2, \ldots, k,
\end{gathered}
$$

where $\nu_{1}, v_{2}$, and $\nu_{3}$ are not integers. By considering $r=4$ and $s=8$, we interpolate (5) at the points $t_{n}, t_{n+1}, t_{n+2}$, and $t_{n+3}$ and collocate (6) at the points $t_{n}, t_{n+1 / 2}, t_{n+1}, t_{n+3 / 2}, t_{n+2}, t_{n+5 / 2}$, $t_{n+3}$, and $t_{n+4}$. This leads to a system of twelve equations which is solved by Mathematica. The values of $\phi_{j}$ are substituted into (4) to develop the multistep method:

$$
Y(t)=\sum_{j=0}^{k} \alpha_{j} y_{n+j}+h^{4}\left(\sum_{j=0}^{k} \beta_{j} f_{n+j}+\sum_{j=1}^{3} \beta_{v_{j}} f_{n+v_{j}}\right),
$$

where $\alpha_{j}, \beta_{j}$, and $\beta_{v_{j}}$ are constant coefficients. Hence, the block hybrid collocation method can be derived as follows.

Main Method. Consider the following

$$
\begin{aligned}
y_{n+4}= & 4 y_{n+3}-6 y_{n+2}+4 y_{n+1}-y_{n}+\frac{h^{4}}{15120} \\
& \times\left(19 f_{n}+1804 f_{n+1}+2560 f_{n+3 / 2}+6354 f_{n+2}\right. \\
& \left.+2560 f_{n+5 / 2}+1804 f_{n+3}+19 f_{n+4}\right) .
\end{aligned}
$$

Additional Method. Consider the following

$$
\begin{aligned}
y_{n+1 / 2}= & \frac{1}{16} y_{n+3}-\frac{5}{16} y_{n+2}+\frac{15}{16} y_{n+1}+\frac{5}{16} y_{n}+\frac{h^{4}}{7741440} \\
& \times\left(75 f_{n}-25032 f_{n+1 / 2}-122530 f_{n+1}\right. \\
& \quad-107760 f_{n+3 / 2}-43080 f_{n+2}-3880 f_{n+5 / 2} \\
& \left.\quad-198 f_{n+3}+5 f_{n+4}\right), \\
y_{n+3 / 2}= & \frac{1}{16} y_{n+3}+\frac{9}{16} y_{n+2}+\frac{9}{16} y_{n+1}-\frac{1}{16} y_{n}+\frac{h^{4}}{430080} \\
& \times\left(f_{n}+260 f_{n+1 / 2}+2311 f_{n+1}+4936 f_{n+3 / 2}\right. \\
& \left.+2311 f_{n+2}+260 f_{n+5 / 2}+f_{n+3}\right), \\
y_{n+5 / 2}= & \frac{5}{16} y_{n+3}+\frac{15}{16} y_{n+2}-\frac{5}{16} y_{n+1}+\frac{1}{16} y_{n}-\frac{h^{4}}{7741440} \\
& \times\left(23 f_{n}+4680 f_{n+1 / 2}+41330 f_{n+1}\right. \\
& +110000 f_{n+3 / 2}+120780 f_{n+2}+25832 f_{n+5 / 2} \\
& \left.\quad-250 f_{n+3}+5 f_{n+4}\right) .
\end{aligned}
$$

The general fourth order differential equations involve the first, second, and third derivatives. In order to generate the formula for the derivatives, the values of $\phi_{j}$ are substituted into

$$
\begin{aligned}
Y^{\prime}(t) & =\sum_{j=1}^{r+s-1} j \phi_{j} t^{j-1}, \\
Y^{\prime \prime}(t) & =\sum_{j=2}^{r+s-1} j(j-1) \phi_{j} t^{j-2}, \\
Y^{\prime \prime \prime}(t) & =\sum_{j=3}^{r+s-1} j(j-1)(j-2) \phi_{j} t^{j-3} .
\end{aligned}
$$

This is obtained by imposing that

$$
\begin{aligned}
Y^{\prime}(t)=\frac{1}{h}\left(\sum_{j=0}^{k} \alpha_{j}^{\prime} y_{n+j}+h^{4}\right. & \\
& \left.\times\left(\sum_{j=0}^{k} \beta_{j}^{\prime} f_{n+j}+\sum_{j=1}^{3} \beta_{v_{j}}^{\prime} f_{n+v_{j}}\right)\right), \\
Y^{\prime \prime}(t)=\frac{1}{h^{2}}\left(\sum_{j=0}^{k} \alpha_{j}^{\prime \prime} y_{n+j}+h^{4}\right. & \\
& \left.\times\left(\sum_{j=0}^{k} \beta_{j}^{\prime \prime} f_{n+j}+\sum_{j=1}^{3} \beta_{v_{j}}^{\prime \prime} f_{n+v_{j}}\right)\right),
\end{aligned}
$$


TABLE 1: Coefficients $\alpha_{i}^{\prime}$ and $\beta_{i}^{\prime}$ for the method (11) evaluated at $t_{n+i / 2}$ for $i=0,1, \ldots, 6$ and $t_{n+4}$.

\begin{tabular}{lccccccccccccccccc}
\hline$t$ & $y_{n}$ & $y_{n+1}$ & $y_{n+2}$ & $y_{n+3}$ & $f_{n}$ & $f_{n+1 / 2}$ & $f_{n+1}$ & $f_{n+3 / 2}$ & $f_{n+2}$ & $f_{n+5 / 2}$ & $f_{n+3}$ & $f_{n+4}$ \\
\hline$t_{n}$ & $-\frac{11}{6}$ & 3 & $-\frac{3}{2}$ & $\frac{1}{3}$ & $-\frac{3397}{3326400}$ & $-\frac{146}{3465}$ & $-\frac{16201}{166320}$ & $-\frac{116}{1485}$ & $-\frac{3089}{110880}$ & $-\frac{26}{7425}$ & $\frac{1}{23760}$ & $-\frac{1}{665280}$ \\
$t_{n+1 / 2}$ & $-\frac{23}{24}$ & $\frac{7}{8}$ & $\frac{1}{8}$ & $-\frac{1}{24}$ & $\frac{7}{12165120}$ & $\frac{114727}{13305600}$ & $\frac{636767}{31933440}$ & $\frac{2461}{295680}$ & $\frac{28903}{6082560}$ & $-\frac{127}{1995840}$ & $\frac{179}{1900800}$ & $-\frac{593}{255467520}$ \\
$t_{n+1}$ & $-\frac{1}{3}$ & $-\frac{1}{2}$ & 1 & $-\frac{1}{6}$ & $\frac{127}{9979200}$ & $\frac{113}{34650}$ & $\frac{13613}{498960}$ & $\frac{5827}{155925}$ & $\frac{503}{36960}$ & $\frac{569}{311850}$ & $-\frac{71}{2494800}$ & $\frac{1}{1425600}$ \\
$t_{n+3 / 2}$ & $\frac{1}{24}$ & $-\frac{9}{8}$ & $\frac{9}{8}$ & $-\frac{1}{24}$ & $-\frac{197}{17031168}$ & $-\frac{13}{42240}$ & $-\frac{4231}{1064448}$ & $-\frac{409}{1900800}$ & $\frac{4079}{946176}$ & $\frac{41}{266112}$ & $\frac{481}{10644480}$ & $-\frac{409}{425779200}$ \\
$t_{n+2}$ & $\frac{1}{6}$ & -1 & $\frac{1}{2}$ & $\frac{1}{3}$ & $\frac{13}{3326400}$ & $-\frac{89}{51975}$ & $-\frac{6913}{498960}$ & $-\frac{214}{5775}$ & $-\frac{9157}{332640}$ & $-\frac{491}{155925}$ & $-\frac{31}{831600}$ & $\frac{1}{1425600}$ \\
$t_{n+5 / 2}$ & $\frac{1}{24}$ & $-\frac{1}{8}$ & $-\frac{7}{8}$ & $\frac{23}{24}$ & $-\frac{2359}{182476800}$ & $-\frac{13}{42240}$ & $-\frac{17971}{4561920}$ & $-\frac{74749}{7983360}$ & $-\frac{271477}{14192640}$ & $-\frac{179503}{19958400}$ & $\frac{23}{285120}$ & $-\frac{593}{255467520}$ \\
$t_{n+3}$ & $-\frac{1}{3}$ & $\frac{3}{2}$ & -3 & $\frac{11}{6}$ & $\frac{1}{95040}$ & $\frac{113}{34650}$ & $\frac{4721}{166320}$ & $\frac{23}{297}$ & $\frac{10859}{110880}$ & $\frac{871}{20790}$ & $\frac{893}{831600}$ & $-\frac{1}{665280}$ \\
$t_{n+4}$ & $-\frac{11}{6}$ & 7 & $-\frac{19}{2}$ & $\frac{13}{3}$ & $\frac{2159}{285120}$ & $-\frac{146}{3465}$ & $\frac{36541}{99792}$ & $\frac{2956}{155925}$ & $\frac{3697}{3168}$ & $\frac{2918}{31185}$ & $\frac{33047}{71280}$ & $\frac{85741}{9979200}$ \\
\hline
\end{tabular}

TABle 2: Coefficients $\alpha_{i}^{\prime \prime}$ and $\beta_{i}^{\prime \prime}$ for the method (12) evaluated at $t_{n+i / 2}$ for $i=0,1, \ldots, 6$ and $t_{n+4}$.

\begin{tabular}{lcccccccccccccccc}
\hline$t$ & $y_{n}$ & $y_{n+1}$ & $y_{n+2}$ & $y_{n+3}$ & $f_{n}$ & $f_{n+1 / 2}$ & $f_{n+1}$ & $f_{n+3 / 2}$ & $f_{n+2}$ & $f_{n+5 / 2}$ & $f_{n+3}$ & $f_{n+4}$ \\
\hline$t_{n}$ & 2 & -5 & 4 & -1 & $\frac{1597}{100800}$ & $\frac{169}{675}$ & $\frac{4439}{15120}$ & $\frac{446}{1575}$ & $\frac{1601}{30240}$ & $\frac{37}{1575}$ & $-\frac{71}{25200}$ & $\frac{23}{302400}$ \\
$t_{n+1 / 2}$ & $\frac{3}{2}$ & $-\frac{7}{2}$ & $\frac{5}{2}$ & $-\frac{1}{2}$ & $-\frac{1973}{3225600}$ & $\frac{107}{19200}$ & $\frac{1933}{15120}$ & $\frac{65911}{604800}$ & $\frac{14927}{322560}$ & $\frac{437}{134400}$ & $\frac{439}{1209600}$ & $-\frac{13}{1382400}$ \\
$t_{n+1}$ & 1 & -2 & 1 & 0 & $\frac{1}{12096}$ & $-\frac{107}{9450}$ & $-\frac{185}{3024}$ & $-\frac{7}{675}$ & $-\frac{29}{30240}$ & $\frac{1}{1890}$ & $-\frac{1}{8400}$ & $\frac{1}{302400}$ \\
$t_{n+3 / 2}$ & $\frac{1}{2}$ & $-\frac{1}{2}$ & $-\frac{1}{2}$ & $\frac{1}{2}$ & $-\frac{59}{2419200}$ & $-\frac{5753}{1209600}$ & $-\frac{4253}{96768}$ & $-\frac{13411}{120960}$ & $-\frac{4253}{96768}$ & $-\frac{5753}{1209600}$ & $-\frac{59}{2419200}$ & 0 \\
$t_{n+2}$ & 0 & 1 & -2 & 1 & $-\frac{1}{302400}$ & 0 & $\frac{1}{5040}$ & $-\frac{8}{675}$ & $-\frac{121}{2016}$ & $-\frac{8}{675}$ & $\frac{1}{5040}$ & $-\frac{1}{302400}$ \\
$t_{n+5 / 2}$ & $-\frac{1}{2}$ & $\frac{5}{2}$ & $-\frac{7}{2}$ & $\frac{3}{2}$ & $\frac{109}{3225600}$ & $\frac{5753}{1209600}$ & $\frac{10399}{241920}$ & $\frac{68459}{604800}$ & $\frac{120527}{967680}$ & $\frac{1223}{172800}$ & $-\frac{569}{604800}$ & $\frac{5}{1382400}$ \\
$t_{n+3}$ & -1 & 4 & -5 & 2 & $-\frac{47}{302400}$ & $\frac{107}{9450}$ & $\frac{401}{5040}$ & $\frac{1177}{4725}$ & $\frac{9683}{30240}$ & $\frac{2251}{9450}$ & $\frac{139}{75600}$ & $-\frac{13}{302400}$ \\
$t_{n+4}$ & -2 & 7 & -8 & 3 & $\frac{10247}{302400}$ & $-\frac{169}{675}$ & $\frac{1121}{1008}$ & $-\frac{146}{105}$ & $\frac{18653}{6048}$ & $-\frac{1781}{1575}$ & $\frac{106483}{75600}$ & $\frac{143}{2880}$ \\
\hline
\end{tabular}

$$
\begin{aligned}
Y^{\prime \prime \prime}(t)=\frac{1}{h^{3}}( & \sum_{j=0}^{k} \alpha_{j}^{\prime \prime \prime} y_{n+j}+h^{4} \\
& \left.\times\left(\sum_{j=0}^{k} \beta_{j}^{\prime \prime \prime} f_{n+j}+\sum_{j=1}^{3} \beta_{v_{j}}^{\prime \prime \prime} f_{n+v_{j}}\right)\right) .
\end{aligned}
$$

The formula for the first, second, and third derivatives is depicted in Tables 1, 2, and 3, respectively.

\section{Order and Stability Properties}

Following the idea of Henrici [16] and Jator [2, 14], the linear difference operator associated with (3) is defined as

$$
\begin{aligned}
L[y(t) ; h]= & \sum_{j=0}^{k}\left[\alpha_{j} y(t+j h)-h^{4} \beta_{j} y^{(4)}(t+j h)\right] \\
& +\sum_{j=1}^{3}\left[\alpha_{v_{j}} y\left(t+v_{j} h\right)-h^{4} \beta_{v_{j}} y^{(4)}\left(t+v_{j} h\right)\right],
\end{aligned}
$$


TABLE 3: Coefficients $\alpha_{i}^{\prime \prime \prime}$ and $\beta_{i}^{\prime \prime \prime}$ for the method (13) evaluated at $t_{n+i / 2}$ for $i=0,1, \ldots, 6$ and $t_{n+4}$.

\begin{tabular}{cccccccccccccccccc}
\hline$t$ & $y_{n}$ & $y_{n+1}$ & $y_{n+2}$ & $y_{n+3}$ & $f_{n}$ & $f_{n+1 / 2}$ & $f_{n+1}$ & $f_{n+3 / 2}$ & $f_{n+2}$ & $f_{n+5 / 2}$ & $f_{n+3}$ & $f_{n+4}$ \\
\hline$t_{n}$ & -1 & 3 & -3 & 1 & $-\frac{33569}{226800}$ & $-\frac{2357}{3150}$ & $-\frac{275}{4536}$ & $-\frac{8747}{14175}$ & $\frac{61}{360}$ & $-\frac{3317}{28350}$ & $\frac{2507}{113400}$ & $-\frac{19}{32400}$ \\
$t_{n+1 / 2}$ & -1 & 3 & -3 & 1 & $\frac{139673}{29030400}$ & $-\frac{35453}{201600}$ & $-\frac{789907}{1451520}$ & $-\frac{134153}{907200}$ & $-\frac{9473}{64512}$ & $\frac{26767}{1814400}$ & $-\frac{5101}{1036800}$ & $\frac{3601}{29030400}$ \\
$t_{n+1}$ & -1 & 3 & -3 & 1 & $-\frac{239}{226800}$ & $\frac{73}{3150}$ & $-\frac{739}{5670}$ & $-\frac{4577}{14175}$ & $-\frac{61}{1260}$ & $-\frac{647}{28350}$ & $\frac{34}{14175}$ & $-\frac{13}{226800}$ \\
$t_{n+3 / 2}$ & -1 & 3 & -3 & 1 & $\frac{14393}{29030400}$ & $\frac{1027}{201600}$ & $\frac{160589}{1451520}$ & $\frac{1201}{129600}$ & $-\frac{40357}{322560}$ & $\frac{2767}{1814400}$ & $-\frac{14107}{7257600}$ & $\frac{1201}{29030400}$ \\
$t_{n+2}$ & -1 & 3 & -3 & 1 & $-\frac{89}{226800}$ & $\frac{43}{3150}$ & $\frac{1553}{22680}$ & $\frac{4213}{14175}$ & $\frac{379}{2520}$ & $-\frac{131}{4050}$ & $\frac{347}{113400}$ & $-\frac{13}{226800}$ \\
$t_{n+5 / 2}$ & -1 & 3 & -3 & 1 & $\frac{2399}{4147200}$ & $\frac{1027}{201600}$ & $\frac{30025}{290304}$ & $\frac{184567}{907200}$ & $\frac{161531}{322560}$ & $\frac{355087}{1814400}$ & $-\frac{66427}{7257600}$ & $\frac{3601}{29030400}$ \\
$t_{n+3}$ & -1 & 3 & -3 & 1 & $-\frac{359}{226800}$ & $\frac{73}{3150}$ & $\frac{29}{810}$ & $\frac{5023}{14175}$ & $\frac{67}{252}$ & $\frac{18553}{28350}$ & $\frac{2389}{14175}$ & $-\frac{19}{32400}$ \\
$t_{n+4}$ & -1 & 3 & -3 & 1 & $\frac{21631}{226800}$ & $-\frac{2357}{3150}$ & $\frac{61601}{22680}$ & $-\frac{70187}{14175}$ & $\frac{17131}{2520}$ & $-\frac{126197}{28350}$ & $\frac{45341}{16200}$ & $\frac{55067}{226800}$ \\
\hline
\end{tabular}

where $y(t)$ is an arbitrary function that is sufficiently differentiable. Expanding the test functions $y(t+j h)$ and $y^{(4)}(t+j h)$ about $t$ and collecting the terms we obtain

$$
L[y(x) ; h]=C_{0} y(x)+C_{1} h y^{\prime}(x)+\cdots+C_{q} h^{q} y^{(q)}(x)+\cdots
$$

whose coefficients $C_{q}$ for $q=0,1, \ldots$ are constants and given as

$$
\begin{aligned}
& C_{0}=\sum_{j=0}^{k} \alpha_{j}+\sum_{j=1}^{3} \alpha_{v_{j}}, \\
& C_{1}=\sum_{j=1}^{k} j \alpha_{j}+\sum_{j=1}^{3} v_{j} \alpha_{v_{j}}, \\
& \vdots \\
& C_{q}=\frac{1}{q !}\left[\sum_{j=1}^{k} j^{q} \alpha_{j}+\sum_{j=1}^{3} v_{j}^{q} \alpha_{v_{j}}-q(q-1)\right. \\
& \left.\quad \times(q-2)(q-3)\left(\sum_{j=1}^{k} j^{q-4} \beta_{j}+\sum_{j=1}^{3} v_{j}^{q-4} \beta_{v_{j}}\right)\right] .
\end{aligned}
$$

According to Jator [2], the linear multistep method is said to be of order $p$ if

$$
C_{0}=C_{1}=\cdots=C_{p+3}=0, \quad C_{p+4} \neq 0 .
$$

The main method (8) and the additional methods (9) are the order eight methods with the error constants; $C_{12}$ are $-1 / 207360,-13 / 679477248,-1 / 7927234560$, and $29 / 4756340736$, respectively. With the order $p>1$, we stipulate the consistency of the method (see $[2,16])$.
In the sense of Jator [2], the hybrid methods (8)-(9) are normalized in block form to analyze the zero stability. The first characteristic polynomial is defined as

$$
\rho(z)=\operatorname{Det}\left[z A^{(0)}-A^{(1)}\right]=z^{6}(z-1)
$$

with

$$
A^{(0)}=7 \times 7 \text { Identity matrix, }
$$

$$
A^{(1)}=\left[\begin{array}{rrrrrrr}
0 & -\frac{5}{4} & 0 & -\frac{5}{2} & 0 & -\frac{10}{3} & \frac{5}{16} \\
0 & \frac{22}{3} & 0 & \frac{44}{3} & 0 & \frac{176}{9} & -\frac{11}{6} \\
0 & \frac{1}{4} & 0 & \frac{1}{2} & 0 & \frac{2}{3} & -\frac{1}{16} \\
0 & -8 & 0 & -16 & 0 & -\frac{64}{3} & 2 \\
0 & -\frac{1}{4} & 0 & -\frac{1}{2} & 0 & -\frac{2}{3} & \frac{1}{16} \\
0 & 4 & 0 & 8 & 0 & \frac{32}{3} & -1 \\
0 & 4 & 0 & 8 & 0 & \frac{32}{3} & -1
\end{array}\right] .
$$

Since the roots of (18) satisfy $\left|z_{i}\right| \leq 1$ for $i=1,2, \ldots, 7$, the method is zero stable.

\section{Numerical Experiment}

The following problems are solved numerically to illustrate the efficiency of the block hybrid collocation method. 
TABLE 4: Numerical results for Problem 1.

\begin{tabular}{lcc}
\hline$h$ & Method & Absolute error at $t=2$ \\
\hline \multirow{3}{*}{0.1} & BHCM4 & $1.74(-8)$ \\
& Adams & $2.11(-3)$ \\
& Jator & $1.26(-4)$ \\
\hline \multirow{3}{*}{0.05} & BHCM4 & $8.45(-11)$ \\
& Adams & $5.37(-4)$ \\
& Jator & $1.91(-6)$ \\
\hline \multirow{3}{*}{0.025} & BHCM4 & $3.69(-13)$ \\
& Adams & $5.09(-5)$ \\
& Jator & $2.96(-8)$ \\
\hline \multirow{3}{*}{0.02} & BHCM4 & $7.11(-14)$ \\
& Adams & $2.25(-5)$ \\
& Jator & $8.65(-9)$
\end{tabular}

Problem 1. Consider the linear fourth order problem (see [2]):

$$
\begin{aligned}
& y^{(4)}=y^{\prime \prime \prime}+y^{\prime \prime}+y^{\prime}+2 y, \quad 0 \leq t \leq 2, \\
& y(0)=y^{\prime}(0)=y^{\prime \prime}(0)=0, \quad y^{\prime \prime \prime}(0)=30,
\end{aligned}
$$

and theoretical solution: $y(t)=2 e^{2 t}-5 e^{-t}+3 \cos t-9 \sin t$.

Problem 2. Consider the nonlinear fourth order problem (see [9]):

$$
\begin{aligned}
& y^{(4)}=\left(y^{\prime}\right)^{2}-y y^{\prime \prime \prime}-4 t^{2}+e^{t}\left(1+t^{2}-4 t\right), \quad 0 \leq t \leq 1, \\
& y(0)=y^{\prime}(0)=1, \quad y^{\prime \prime}(0)=3, \quad y^{\prime \prime \prime}(0)=1,
\end{aligned}
$$

and theoretical solution: $y(t)=t^{2}+e^{t}$.

The block hybrid collocation method is implemented together with the Mathematica built-in packages, namely, Solve and FindRoot for the solution of linear and nonlinear problems, respectively.

The performance comparison between block hybrid collocation method with the existing methods $[2,9]$ and the Adams Bashforth-Adams Moulton method is presented in Tables 4 and 5. The following notations are used in the tables:

\section{$h$ : step size;}

BHCM4: block hybrid collocation method;

Adams: Adams Bashforth-Adams Moulton method;

Awoyemi: multiderivative collocation method in Awoyemi [9];

Jator: finite difference method in Jator [2].

Tables 4 and 5 show the superiority of BHCM4 in terms of accuracy over the existing Adams method, Jator finite difference method [2], and Awoyemi multiderivative collocation method [9].
TABle 5: Numerical results for Problem 2.

\begin{tabular}{lcc}
\hline$h$ & Method & Absolute error at $t=1$ \\
\hline \multirow{3}{*}{0.2} & BHCM4 & $2.38(-12)$ \\
& Adams & $5.01(-7)$ \\
& Awoyemi & $5.84(-4)$ \\
\hline \multirow{3}{*}{0.1} & BHCM4 & $1.95(-14)$ \\
& Adams & $2.44(-6)$ \\
& Awoyemi & $9.26(-5)$ \\
\hline
\end{tabular}

TABle 6: Performance comparison for Wu equation with $\epsilon=0$.

\begin{tabular}{lcc}
\hline$h$ & Method & Absolute error at $t=15$ \\
\hline \multirow{3}{*}{0.25} & BHCM4 & $5.2(-7)$ \\
& Adams & $4.9(-3)$ \\
& Twizell & $1.9(-4)$ \\
\hline \multirow{3}{*}{0.1} & BHCM4 & $2.8(-10)$ \\
& Adams & $8.4(-5)$ \\
& Cortell & $3.7(-5)$ \\
\hline
\end{tabular}

\section{Application to Problem from Ship Dynamics [5-7]}

The proposed method is also applied to solve a physical problem from ship dynamics. As stated by $\mathrm{Wu}$ et al. [5], when a sinusoidal wave of frequency $\Omega$ passes along a ship or offshore structure, the resultant fluid actions vary with time $t$. In a particular case study by Wu et al. [5], the fourth order problem is defined as

$$
y^{(4)}+3 y^{\prime \prime}+y(2+\epsilon \cos (\Omega t))=0, \quad t>0,
$$

which is subjected to the following initial conditions:

$$
y(0)=1, \quad y^{\prime}(0)=y^{\prime \prime}(0)=y^{\prime \prime \prime}(0)=0,
$$

where $\epsilon=0$ for the existence of the theoretical solution, $y(t)=2 \cos t-\cos (t \sqrt{2})$. The theoretical solution is undefined when $\epsilon \neq 0$ (see [6]).

In the literature, some numerical methods for solving fourth order ODEs have been extended to solve the problem from ship dynamics. Numerical investigation was presented in Twizell [6] and Cortell [7] concerning the fourth order $\operatorname{ODEs}(22)$ for the cases $\epsilon=0$ and $\epsilon=1$ with $\Omega=0.25(\sqrt{2}-1)$. Instead of solving the fourth order ODEs directly, Twizell [6] and Cortell [7] considered the conventional approach of reduction to system of first order ODEs. Twizell [6] developed a family of numerical methods with the global extrapolation to increase the order of the methods. On the other hand, Cortell [7] proposed the extension of the classical RungeKutta method.

Table 6 shows the comparison in terms of accuracy for $y$ at the end point $t=15$. BHCM4 manages to achieve better accuracy compared to Adams Bashforth-Adams Moulton method, Twizell [6], and Cortell [7] when $h=0.25$ and $h=0.1$, respectively.

Figure 1 depicts the numerical solution for $\mathrm{Wu}$ equation (22) with $\epsilon=1$ and $\Omega=0.25(\sqrt{2}-1)$ in the interval 


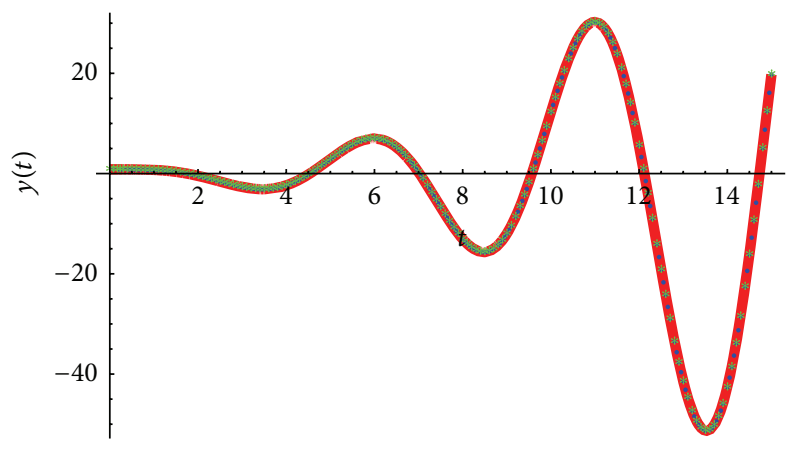

- $\quad$ BHCM4, $h=0.1$

* Cortell, $h=0.1$

NDSolve

Figure 1: Response curve for Wu equation with $\epsilon=1, \Omega=$ $0.25(\sqrt{2}-1)$.

$[0,15]$. The solutions obtained by BHCM4 are in agreement with the observation of Cortell [7] and Mathematica built-in package NDSolve.

\section{Conclusion}

As indicated in the numerical results, the block hybrid collocation method has significant improvement over the existing methods. Furthermore, it is applicable for the solution of physical problem from ship dynamics.

As a conclusion, the block hybrid collocation method is proposed for the direct solution of general fourth order ODEs whereby it is implemented as self-starting method that generates the solution of $y$ at four main points and three offstep points concurrently.

\section{Conflict of Interests}

The authors declare that there is no conflict of interests regarding the publication of this paper.

\section{References}

[1] A. K. Alomari, N. Ratib Anakira, A. S. Bataineh, and I. Hashim, "Approximate solution of nonlinear system of BVP arising in fluid flow problem," Mathematical Problems in Engineering, vol. 2013, Article ID 136043, 7 pages, 2013.

[2] S. N. Jator, "Numerical integrators for fourth order initial and boundary value problems," International Journal of Pure and Applied Mathematics, vol. 47, no. 4, pp. 563-576, 2008.

[3] O. Kelesoglu, "The solution of fourth order boundary value problem arising out of the beam-column theory using Adomian decomposition method," Mathematical Problems in Engineering, vol. 2014, Article ID 649471, 6 pages, 2014.

[4] A. Boutayeb and A. Chetouani, "A mini-review of numerical methods for high-order problems," International Journal of Computer Mathematics, vol. 84, no. 4, pp. 563-579, 2007.

[5] X. J. Wu, Y. Wang, and W. G. Price, "Multiple resonances, responses, and parametric instabilities in offshore structures," Journal of Ship Research, vol. 32, no. 4, pp. 285-296, 1988.
[6] E. H. Twizell, "A family of numerical methods for the solution of high-order general initial value problems," Computer Methods in Applied Mechanics and Engineering, vol. 67, no. 1, pp. 15-25, 1988.

[7] R. Cortell, "Application of the fourth-order Runge-Kutta method for the solution of high-order general initial value problems," Computers and Structures, vol. 49, no. 5, pp. 897-900, 1993.

[8] A. Malek and R. Shekari Beidokhti, "Numerical solution for high order differential equations using a hybrid neural network-optimization method," Applied Mathematics and Computation, vol. 183, no. 1, pp. 260-271, 2006.

[9] D. O. Awoyemi, "Algorithmic collocation approach for direct solution of fourth-order initial-value problems of ordinary differential equations," International Journal of Computer Mathematics, vol. 82, no. 3, pp. 321-329, 2005.

[10] N. Waeleh, Z. A. Majid, F. Ismail, and M. Suleiman, "Numerical solution of higher order ordinary differential equations by direct block code," Journal of Mathematics and Statistics, vol. 8, no. 1, pp. 77-81, 2011.

[11] S. J. Kayode, "An order six zero-stable method for direct solution of fourth order ordinary differential equations," American Journal of Applied Sciences, vol. 5, no. 11, pp. 1461-1466, 2008.

[12] S. J. Kayode, "An efficient zero-stable numerical method for fourth-order differential equations," International Journal of Mathematics and Mathematical Sciences, vol. 2008, Article ID 364021, 10 pages, 2008.

[13] B. T. Olabode and T. J. Alabi, "Direct block predictor-corrector method for the solution of general fourth order ODEs," Journal of Mathematics Research, vol. 5, no. 1, pp. 26-33, 2013.

[14] S. N. Jator, "Solving second order initial value problems by a hybrid multistep method without predictors," Applied Mathematics and Computation, vol. 217, no. 8, pp. 4036-4046, 2010.

[15] L. K. Yap, F. Ismail, and N. Senu, "An accurate block hybrid collocation method for third order ordinary differential equations," Journal of Applied Mathematics, vol. 2014, Article ID 549597, 9 pages, 2014.

[16] P. Henrici, Discrete Variable Methods in Ordinary Differential Equations, John Wiley \& Sons, New York, NY, USA, 1962. 


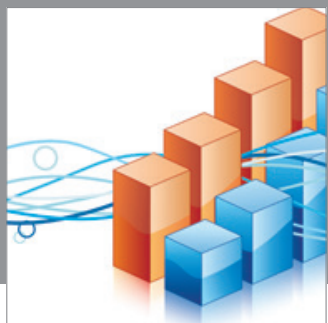

Advances in

Operations Research

mansans

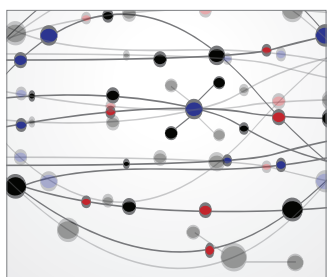

The Scientific World Journal
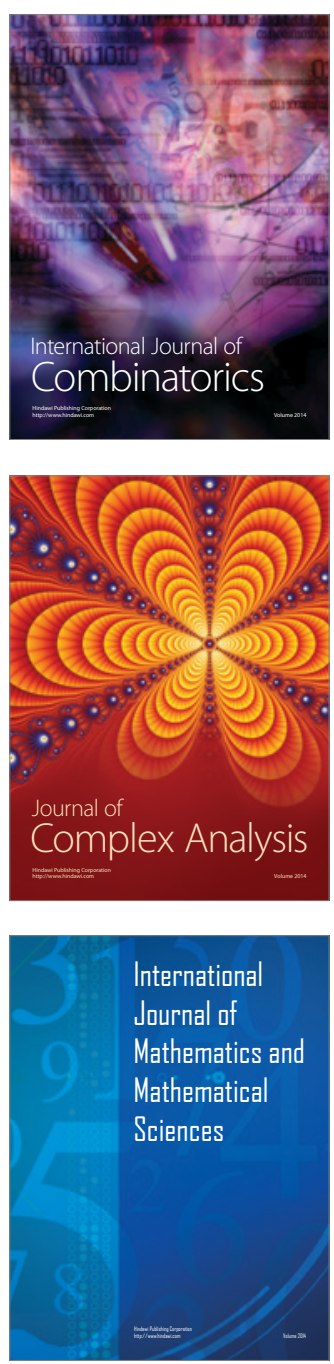
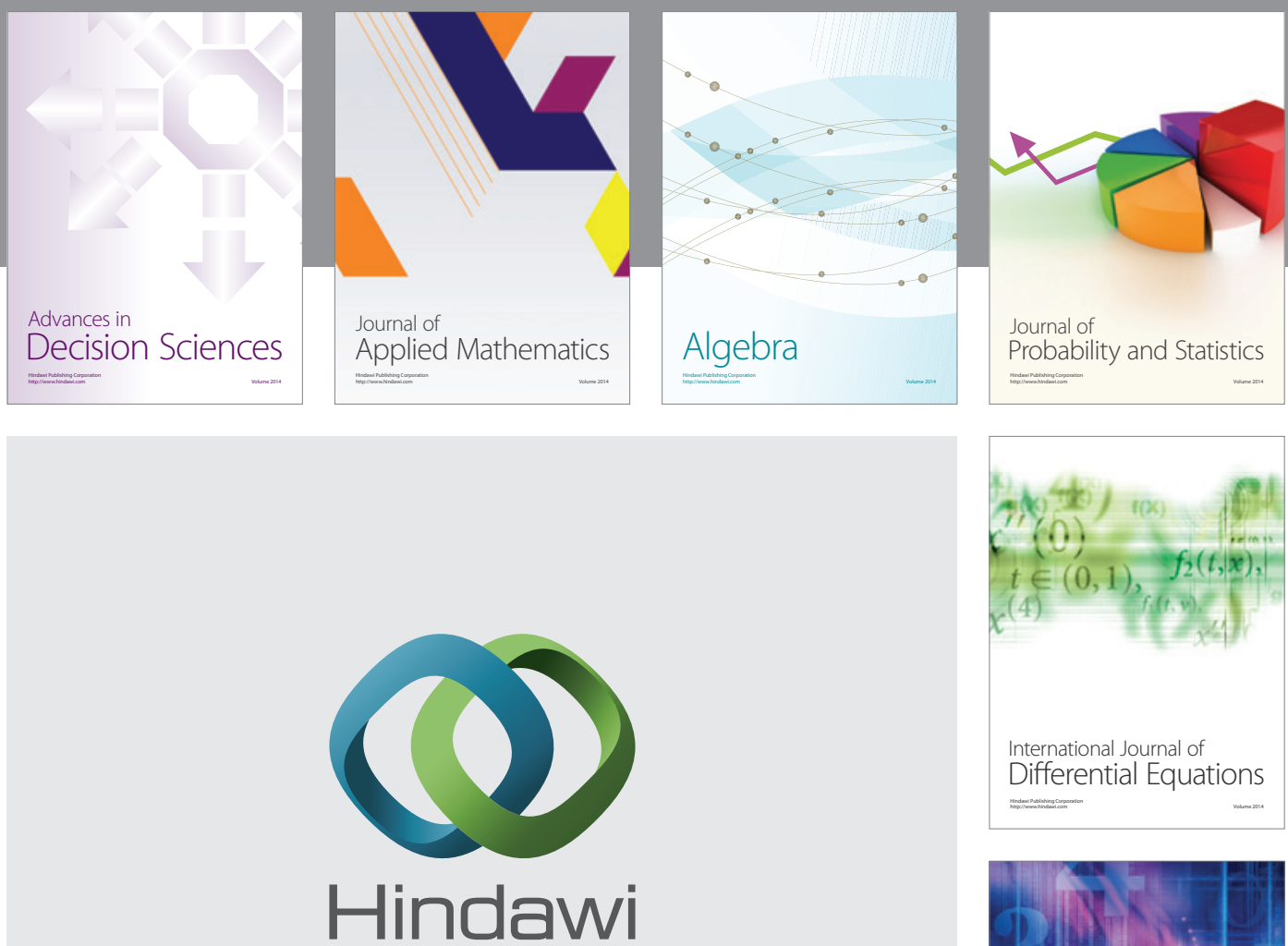

Submit your manuscripts at http://www.hindawi.com
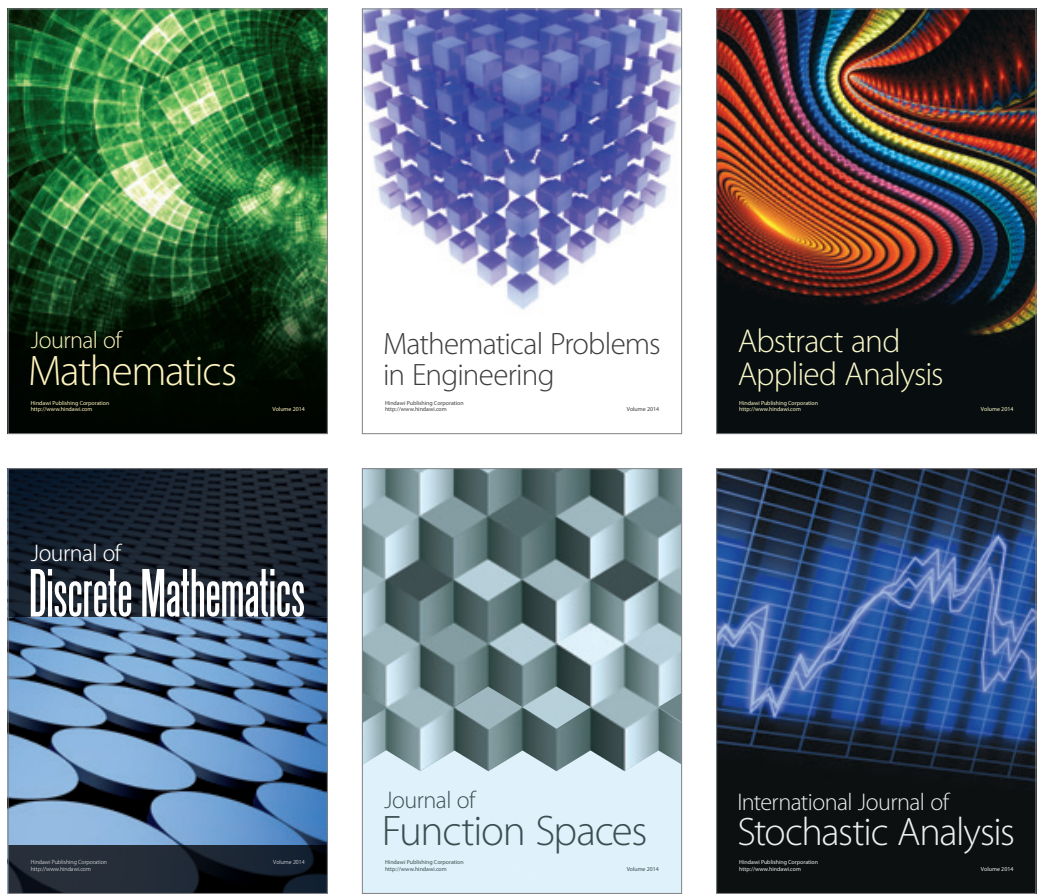

Journal of

Function Spaces

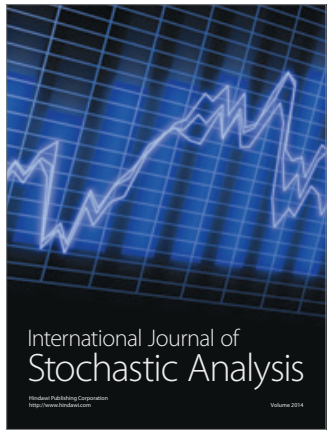

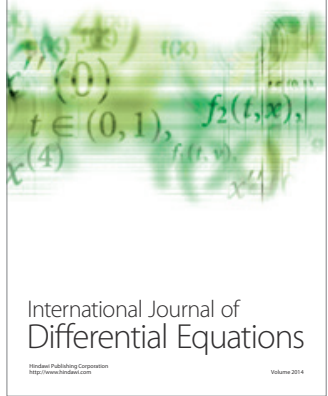
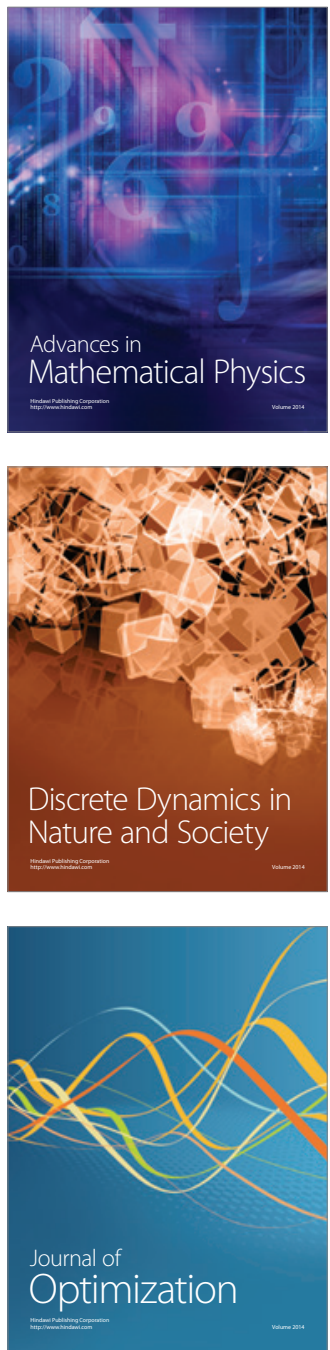